\title{
Influences of Tube Assembly of Wind Turbine Towers with the Steel-Concrete Composite Structure on Towers
}

\author{
Li Chenglin ${ }^{1, a}$, Liu Yanxiang ${ }^{3, b}$, Ma Xinwei ${ }^{2, c, *}$ and Guo Yuancheng ${ }^{1, d}$ \\ ${ }^{1}$ School of Civil Engineering, Zhengzhou University, No. 100, Science Avenue, High-Tect District, \\ Zhengzhou 450001, China \\ ${ }^{2}$ Department of Civil Engineering, Harbn Institute of Technology (Weihai), No. 2, \\ Wenhua West Road, Huancui District, Weihai 264209, China \\ ${ }^{3}$ Hainan Yilong City Construction Investment Co, Ltd, Zuanshi Avenue, Longlou Town, Wenchang 571332, \\ China \\ E-mail: ${ }^{a} 494729589 @ q q . c o m,{ }^{b}$ 1176875158@qq.com, ${ }^{c}$ xinwei.ma@163.com, ${ }^{\text {d }}$ guoyuancheng@163.com \\ *corresponding author
}

Keywords: Steel-concrete composite structure, wind turbine tower, numerical simulation, annular rib, longitudinal rib.

\begin{abstract}
The height of a real wind turbine tower structure is far larger than the length in the plane, so segmented assembly is generally adopted on the spot. However, after assembling a tower, annular ribs for blocking concrete and connecting tubes of a double-layered steel tower in the construction of the tower sections remain in the tower structure. These annular ribs can influence mechanical performances of the tower, so the research is very important. The calculations of finite element models with no ribs and with annular ribs and longitudinal ribs show that the annular ribs used during the segmented assembly slightly affect flexibility of the tower before yielding. However, they have great influences on stress in the inner and outer steel tubes in the compressive zone under the constraints of concrete. Furthermore, concrete slightly constrains longitudinal deformation and shows no obvious effect on flexibility of the tower and stress in the inner and outer steel tubes. The variation of loading direction cannot change the influences of the longitudinal ribs on the tower.
\end{abstract}

\section{Introduction}

With the rapid development of high-power wind turbines, wind turbine tower structures are getting higher and higher. However, the over-high towers are difficult to build, so on-site assembly and segmentation are generally used in the construction of towers. After assembling a tower, the annular ribs for blocking concrete and connecting tubes of the double-layered steel tower in the construction of the tower are left in the tower structure. These ribs can affect mechanical performances of the tower, so the importance of relevant researches is highlighted.

At present, the wind turbine tower structures with horizontal axes are mainly classified into the steel-concrete structure, truss structure and cone shaped steel tower. In 1994, Holmes et al. conducted a dynamic analysis on wind turbine towers. They modeled towers and wind turbines separately by utilizing the modal analysis method and elaborated the influences of coupling relationship of the both on mechanical performances and stability of towers on the whole [1]. Ryan et al. carried out the modal analysis by using the same method, while they researched the impacts of blade flexibility on the coupling effects of towers and wind turbines, thus obtaining reasonable analysis results [2]. Jimeno designed the concrete towers into pre-fabricated arc tower tubes and assembled components of such design on site, which provides guidance for designing huge wind turbine towers [3, 4]. For the purpose of designing commercial wind turbine towers, Qu Chengzhong et al. applied concrete filled double skin steel tube (CFDSST) into wind turbine towers. Based on the dimension parameters of the existing 1.5 MW wind turbine towers with steel 
structures, they improved the wind turbine towers and designed new wind turbine towers with a composite structure. By establishing finite element models through proper simplifications, the mathematical simulation analysis was conducted. The research conclusions demonstrate that such tower structures have high flexural rigidity and strength, and do not resonate with blades [5].

Actually, a wind turbine tower structure has larger size in the height direction than in the plane, so segmented assembly is generally utilized on site. Nevertheless, after assembling the tower, the annular ribs that are used to block concrete and connect tubes of the double-layered steel tower in the construction of the tower sections remain in the tower structure and influence mechanical performances of the tower. By establishing three different models including the tower tubes with no ribs and with annular ribs and longitudinal arc ribs, this study discusses the impacts of the annular and longitudinal ribs used during the segmented assembly on mechanical performances of the tower. The research shows that the annular ribs slightly affect flexibility of the tower before yielding, while have certain influences on stresses in the inner and outer steel tubes in the tower. The longitudinal ribs exert insignificant effects on strengthening deformation resistance of the tower and on stress in the inner and outer steel tubes.

\section{Finite Element Numerical Analysis}

Annular and longitudinal ribs in tower tubes are used to connect the inner and outer steel tubes in the construction and play a supporting role and used as frameworks in concrete casting. After the assembly, it seems that the annular and longitudinal ribs are put into the tower (as shown in Figures 1 and 2). This research studies the influences of the annular and longitudinal ribs used for assembling tower tubes on the tower structure.

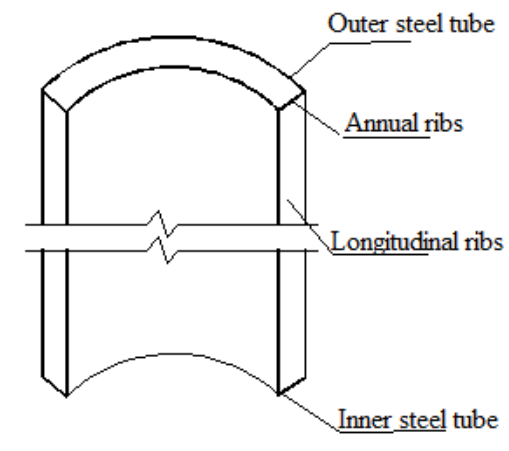

Figure 1 Sketch map of pre-fabricated tower tubes.

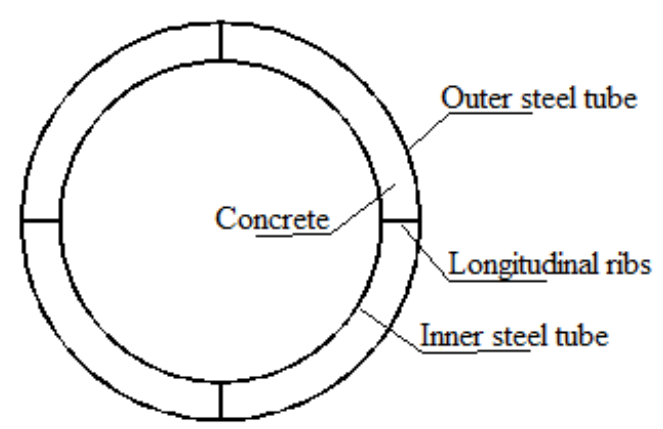

Figure 2 Tower section after assembling.

\subsection{Model Description}

In this study, the abaque finite element analysis software is used for modeling. This study adopts material parameters of steel and concrete obtained from material experiments carried out by Dong Chonghai [6]. Solid elements and shell elements are used to represent the concrete and the steel tubes. Tie contact is used between steels. Moreover, the steels and concrete are hard contact in normal direction and penalty friction contact in the tangent direction, respectively.

Model 1. The model represents a tower tube without ribs in the height of $100 \mathrm{~m}$ and is divided into four sections. The dimensions of the external diameter, internal diameter and thickness of steel tubes are 3,600 mm: 3,150 mm: $15 \mathrm{~mm}$ and 4,800 mm: 4,200 mm: $20 \mathrm{~mm}$ on the top and bottom, respectively.

Model 2. Based on the model 1, the annular ribs are set on three sectional planes between every two adjacent tower sections and the thickness equals to the mean value of thickness of steel tubes in the upper and lower towers.

Model 3. As to the two lower tower sections in the model 2, the tower tubes are equipped with four longitudinal arc plates. As demonstrated in Figure 3, the thickness of the longitudinal ribs is consistent with that of steels in the tower section. 


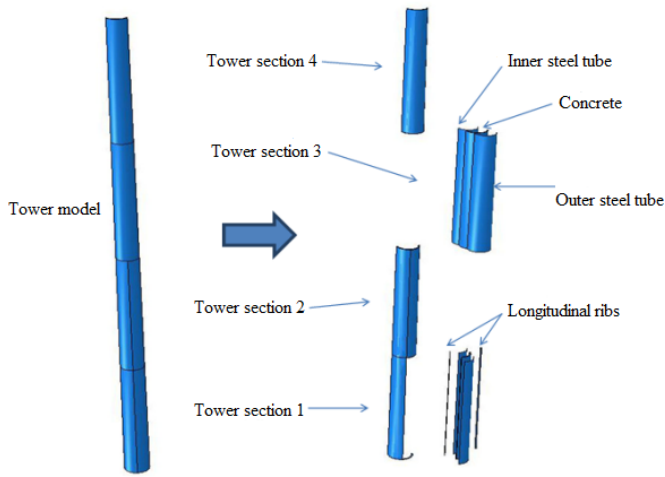

Figure 3 Sketch map of the tower in the model 3.

Differing from that the annular ribs are centred on and symmetrical around $y$ axis, the longitudinal ribs on the section are distributed at intervals. Based on the symmetrical plane of two longitudinal ribs, loads are applied on the model 3 to explore the influences of ribs on the tower when the loading direction is parallel to and shows an angle of $45^{\circ}$ with the symmetrical plane, as displayed in Figure 4. The $\mathrm{x}$ direction is loading direction and the plane passing two longitudinal ribs and the central axis of the tower is regarded as the symmetrical plane. In the figure, it is shown as an axis of symmetry in the section.
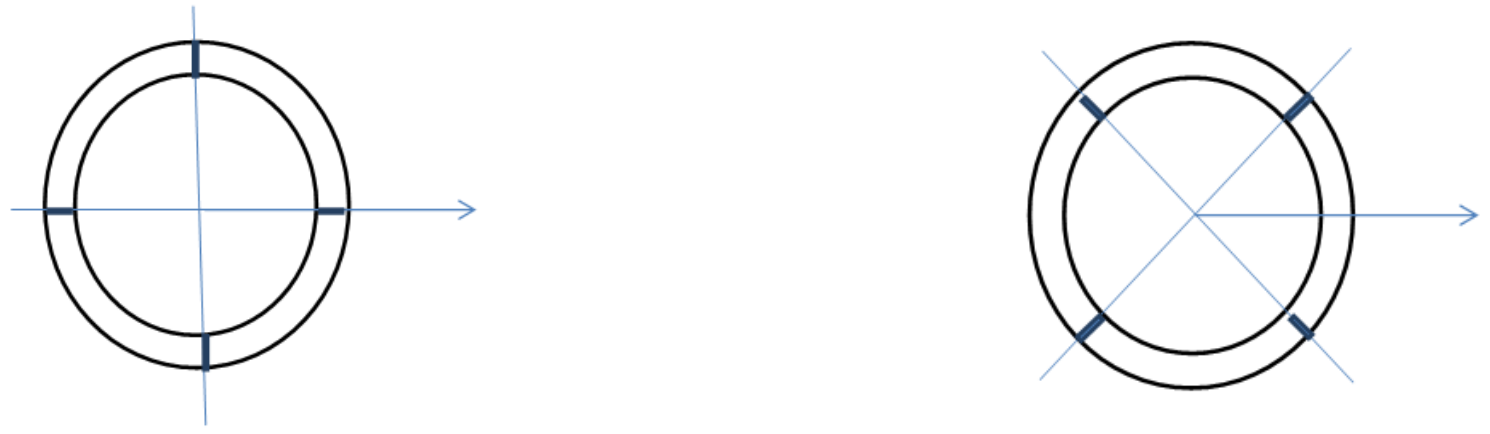

(a) Loading direction being parallel to axis of symmetry.

(b) Loading direction showing an angle of $45^{\circ}$ with the axis of symmetry.

Figure 4 Sketch map of relative position of loading direction and ribs.

\subsection{Impacts of the Annular Ribs on Mechanical Performances of the Tower}

When there is no ribs (model 1) and there are annular ribs (model 2), the curves of changes of horizontal displacement on the top with horizontal loads are displayed in Figure 5. In the elastic stage of linear growth of displacement, the existence of annular ribs slightly reduces the displacement of the tower. After increasing flexibility, that is, after yield stage, the annular ribs begin to show significant weakening effects on the displacement of the tower.

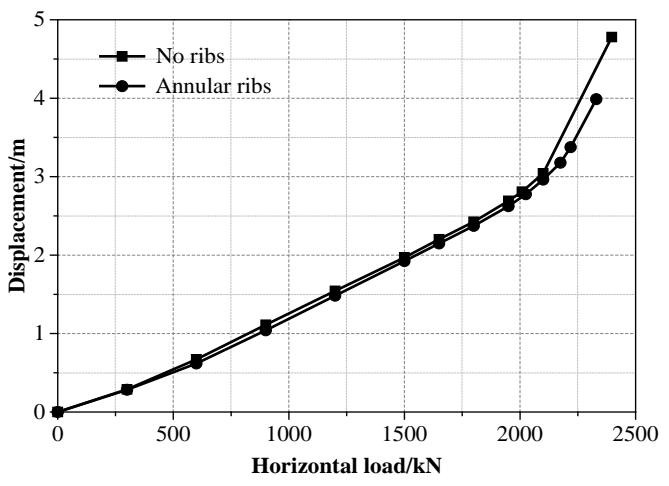

Figure 5 Impacts of the annular ribs on the displacement growth of the tower. 
The reason for the above phenomenon is as follows: The existence of the annular ribs has stronger connection effects on the inner and outer steel tubes than the contact action between steel tubes and concrete. In comparison with the stresses in the tensile and compressed sides of the inner and outer steel tubes in each tower section of the tower tubes without annular rib (model 1) and with annular ribs (model 2), Figure 6 is drawn for analysis.

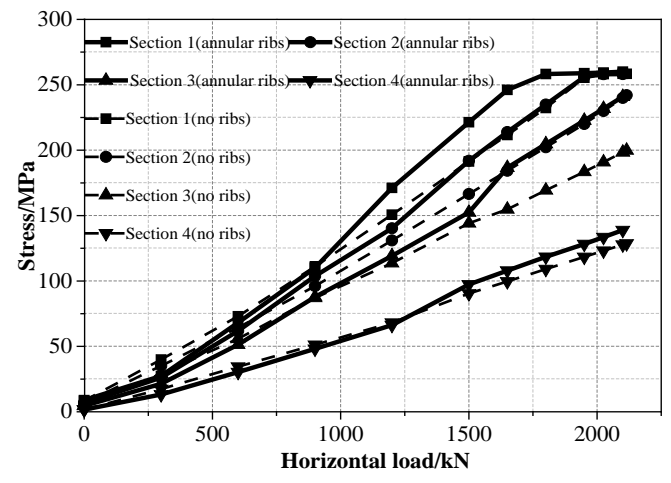

(a) Tensile side of the outer steel tubes.

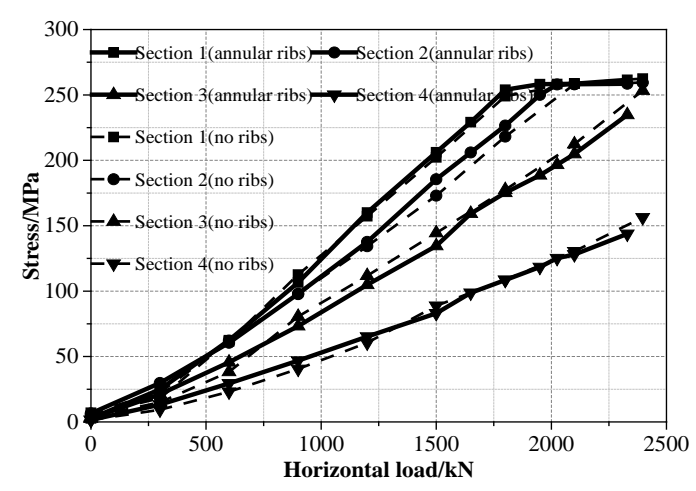

(c) Tensile side of the inner steel tubes.

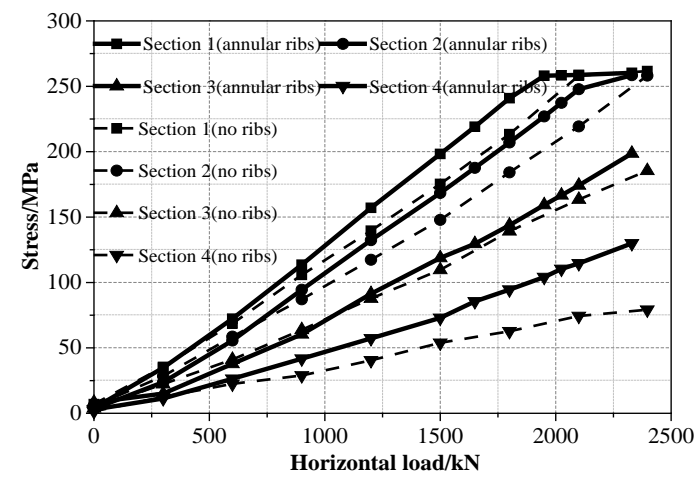

(b) Compressed side of the outer steel tubes.

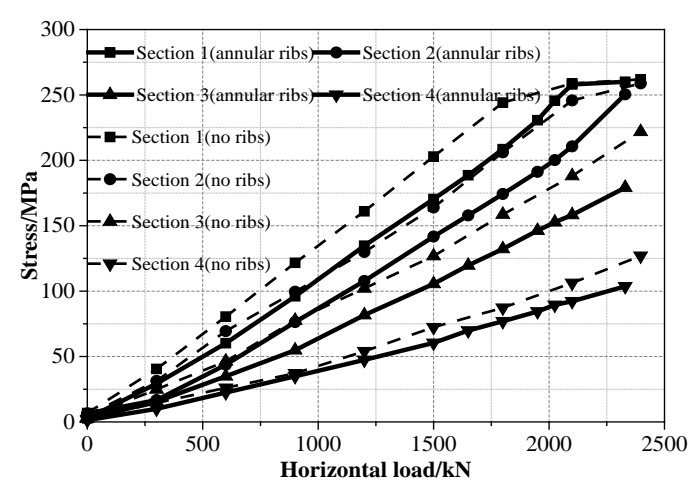

(d) Compressed side of the inner steel tubes.

Figure 6 Influences of setting annular ribs on stresses in different tower sections.

Figures 6(c) and 6(a) show that the presence of the annular ribs has insignificant effects on stress in the tensile side of the inner and outer steel tubes with the increase of loads. Figures 6(b) and 6(d) demonstrate that yield load in the tower section 1 in the outer steel tube decreases from 2,100 kN to $1,900 \mathrm{kN}$ due to the effects of the annular ribs, and the stress in the tower section 4 also raises on the whole. However, in the inner steel tube, stress in each tower section grows slowly because of the existence of annular ribs, and the yield load of the tower sections 1 and 2 also enlarges. The results show that the annular ribs used for assembling tower tubes slightly influence stress in the tensile side. In the compressed side of the outer steel tube, the stress level rises. However, stress level decreases, the growth of stress level slows down and yield load increases in the compressed side of the inner steel tube. This reveals that the annular ribs do not merely play a role in connecting the inner and outer steel tubes.

The reason for such phenomenon appearing is that the compressed side is jointly supported by the inner and outer steel tubes and concrete. When there are no ribs, relative slippage occurs between the steel tubes (inner and outer ones) and concrete, which increases rigidity and reduces stress of the outer steel tube under the internal force. Correspondingly, the internal force in the inner steel tube rises. The annular ribs connecting with the outer steel tube show the same functions with shear key. Moreover, concrete in the compressive zone has certain constraints on ribs, which largely reduces such friction-based slippage, so the stress level in the outer steel tube does not reduce. In the tension zone, even though the inner and outer steel tubes are connected by the annular ribs, such slippage cannot be resisted because concrete supports do not work. The stress nephogram of concrete layers is shown in Figure 7. 


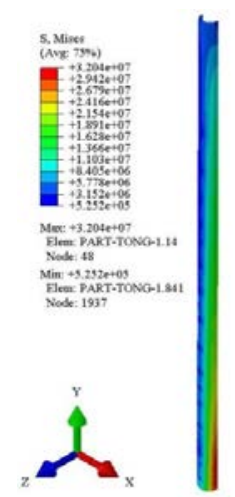

(a) No annular rib

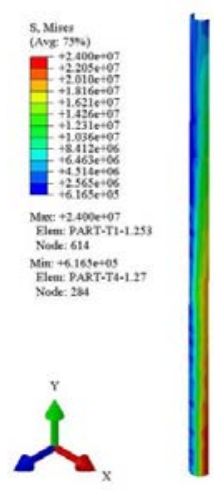

(b) With annular ribs

Figure 7 Stress nephogram in the compressed side of the concrete layers.

It can be seen from the figure that when there is no annular rib, the bottom of the tower is the high stress area of the concrete layers. When annular ribs exist, the high stress area in the concrete layers expands and the stresses distribute more uniformly.

\subsection{Effects of the longitudinal ribs on mechanical performances of the tower}

The changes of displacement with load on conditions that only annular ribs are adopted and that there are longitudinal ribs apart from annular ribs are displayed in Figure 8. The figure shows that the two curves of displacement growth are basically overlapped. When there are only annular ribs and when longitudinal ribs are adopted along with annular ribs, the flexibilities of the tower under the loads before yielding are $1.41 \mathrm{~mm} / \mathrm{kN}$ and $1.38 \mathrm{~mm} / \mathrm{kN}$, respectively, showing a small difference. Therefore, the longitudinal ribs insignificantly affect the displacement growth of the tower before yielding. After the yield area appearing, the flexibilities have some differences and increase to $3.11 \mathrm{~mm} / \mathrm{kN}$ and $2.36 \mathrm{~mm} / \mathrm{kN}$ separately. Therefore, the longitudinal ribs exert small impacts on the displacement of the tower.

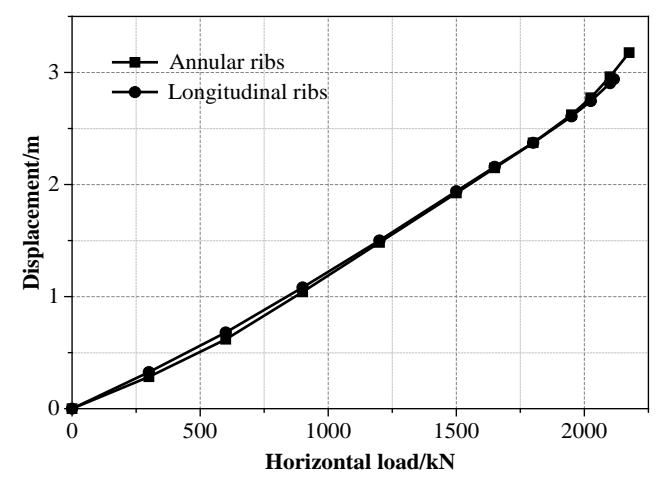

Figure 8 Influences of the longitudinal ribs on the displacement growth of the tower.

The existence of longitudinal ribs does not affect the fact that stress in the tensile side develops faster than that in the compressed side in the outer and inner steel tubes, and that stress in the outer steel tube increases faster than that in the inner steel tube. As demonstrated in Figure 9, when the load is up to $1,800 \mathrm{kN}$, stress reaches to the yield strength in the tensile side of the outer steel tube, while yielding occurs when the load is about $2,000 \mathrm{kN}$ in the compressed side. In the inner steel tube, yielding in the tensile side appears in the tower section 1 when the load reaches to 2,000 kN, while the tower section 1 does not reach to the yield strength in the compressed side before the load reaching to 2,100 $\mathrm{kN}$. No matter in the compressed side or the tensile side, load resulting in yielding in the outer steel tube is smaller than that in the inner steel tube. The stress in the tower when there are the longitudinal ribs together with annular ribs is compared with that in the inner and outer steel tubes in the tower only with the annular ribs. Based on this, although the stress in the inner and outer steel tubes reduces due to the existence of the longitudinal ribs in the tower sections 1 and 2, 
the influence is slight. In the tower sections 3 and 4, owing to the longitudinal ribs exist, stress in the outer steel tube increases.

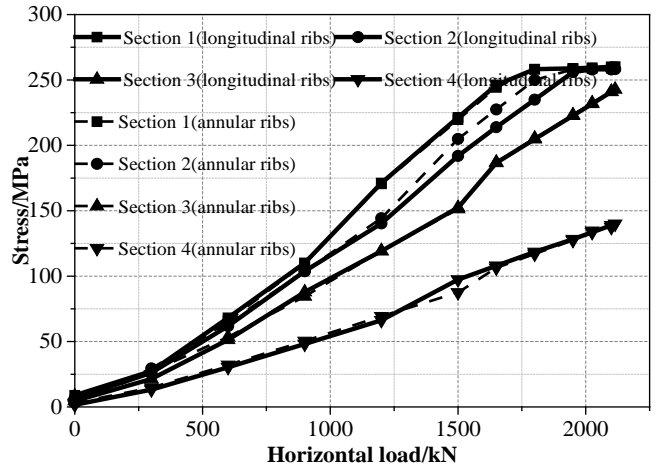

(a) Tensile side of the outer steel tube.

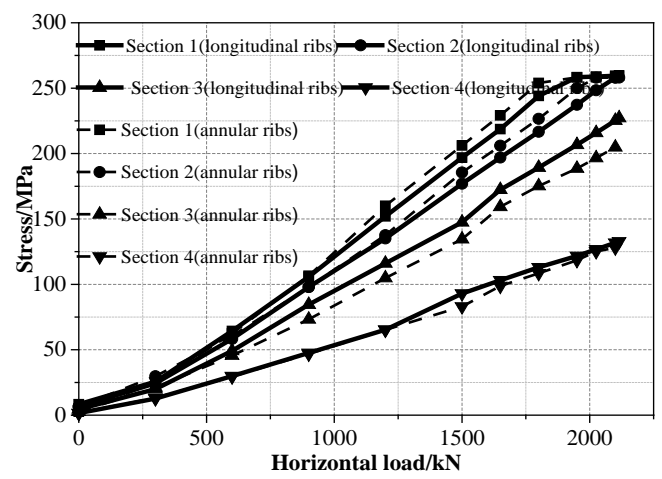

(c) Tensile side of the inner steel tube.

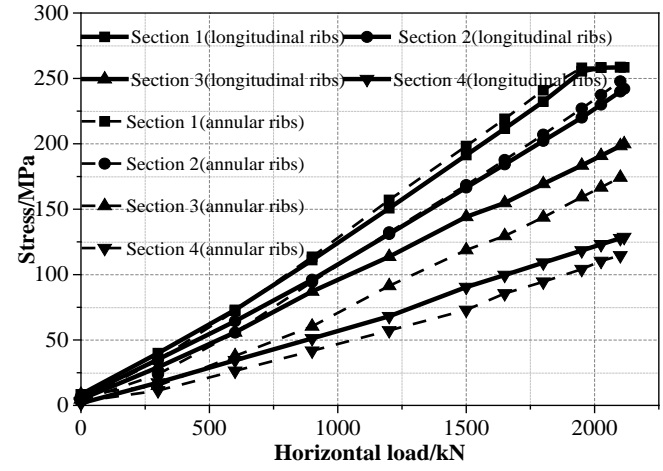

(b) Compressed side of the outer steel tube.

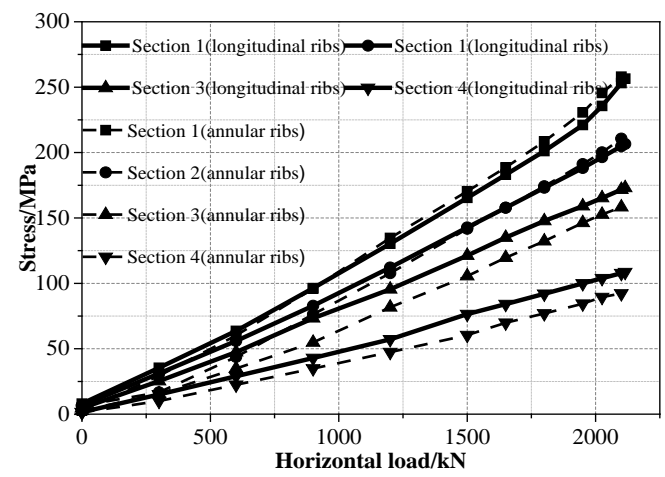

(d) Compressed side of the inner steel tube.

Figure 9 Influences of setting the longitudinal ribs on stress in the lower position of each tower section.

The reasons for small effects of the longitudinal ribs on stress distribution in the inner and outer steel tubes are shown as follows: Firstly, the existence of annular ribs has greatly changed stress distribution in the inner and outer steel tubes, so the longitudinal ribs have small influences on this basis. Secondly, the longitudinal ribs contribute less to the areas of steel on the cross section and therefore have limited capacity in sharing the load. As displayed in Figure 10, the changes of stress in the longitudinal ribs with loads are basically consistent with those in the outer steel tube. Moreover, tangential slippage occurs between the concrete and the longitudinal ribs under the loading effects. Stress nephograms of the concrete layers in the presence of the annular and longitudinal ribs is shown in Figure 11.

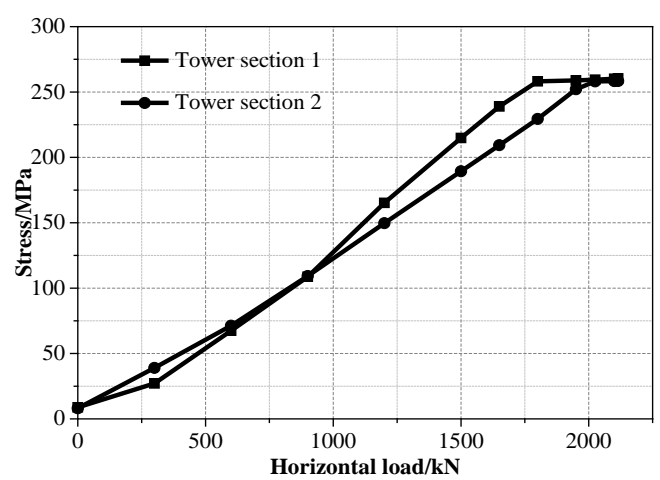

(a) Tensile side

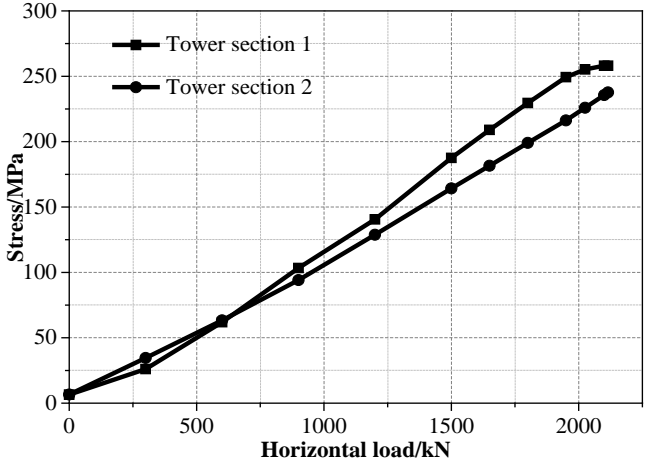

(b) Compressed side

Figure 10 Stress in the longitudinal ribs. 


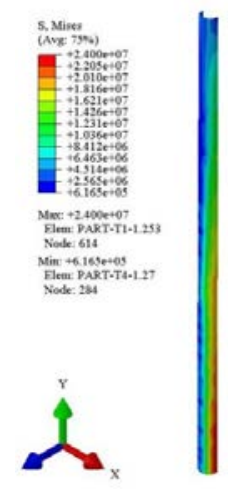

(a) With annular ribs only.

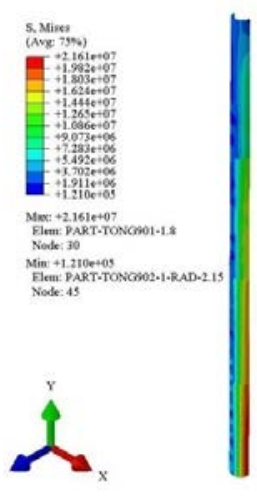

(b) With longitudinal ribs together with annular ribs.

Figure 11 Stress nephograms in the compressed side of the concrete layers when annular and longitudinal ribs exist.

In the finite element simulations, the tangential contact between the longitudinal ribs and concrete layers belongs to Coulomb friction contact. Under the effects of horizontal loads, the tangential slippage occurs. This is different from the fact that normal function mainly appears between the annular ribs and the concrete under the loads. Therefore, the concrete layers have unobvious constraints to the longitudinal ribs. It can be seen from the comparisons of stress nephograms that the stresses in the concrete layers when there are only the annular ribs and when there are both the longitudinal rib and annular ribs distribute extremely closely. This also indirectly verifies that the interaction between the longitudinal ribs and the concrete layers is small.

\subsection{Loading Direction Showing an Angle of $45^{\circ}$ with the Axis of Symmetry of the Longitudinal Ribs}

For the annular ribs, the loading direction is always overlapped with the axis of symmetry no matter how it changes. Different from this, when the loading direction changes, the relative position of the axis of symmetry of the longitudinal ribs and loading direction also changes. Similar to the situation that loads are applied in the loading direction parallel to the ribs, the flexibility of the tower decreases slightly when the load is applied in the direction showing an angle of $45^{\circ}$ with the axis of symmetry of the ribs, as displayed in Figure 12.

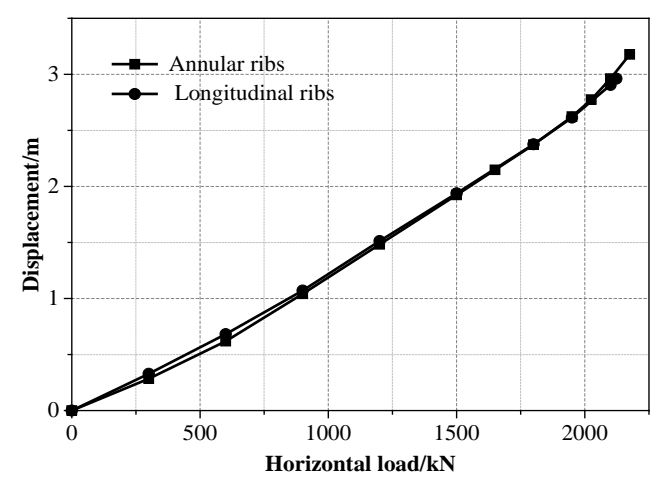

Figure 12 Effects of the longitudinal ribs on displacement growth of the tower.

The comparison of the increases of horizontal displacement on the top of the tower in different loading directions (Figure 13) shows that the two curves are overlapped and the flexibility of the tower is $1.38 \mathrm{~mm} / \mathrm{kN}$. Therefore, the displacement growth of the tower does not change with the relative position of the loading direction and the longitudinal ribs.

When the loading direction demonstrates angles of $0^{\circ}$ and $45^{\circ}$ with the axis of symmetry of the longitudinal ribs, the stresses in the tensile and compressed sides in the inner and outer steel tubes in each tower section are shown in Figure 14. 


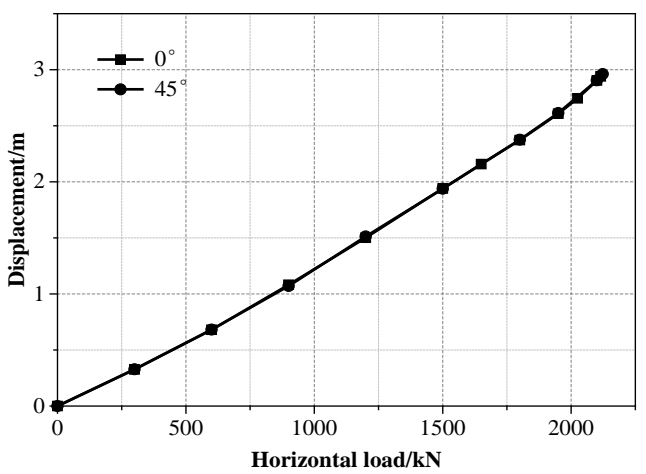

Figure 13 Sketch map of displacement growth of the tower under conditions of different loading directions.

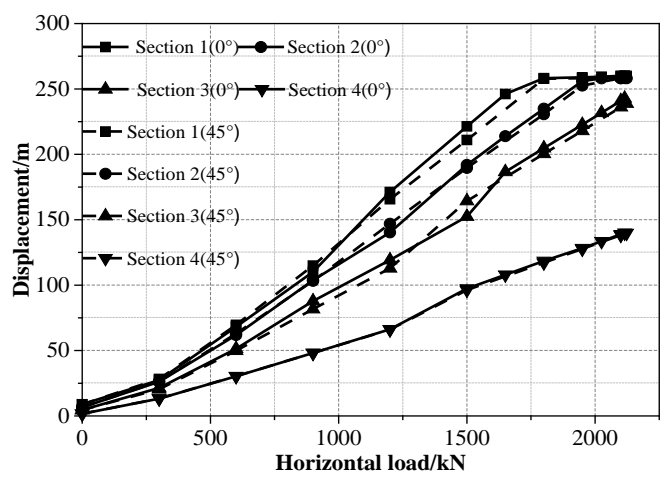

(a) Tensile side of the outer steel tube.

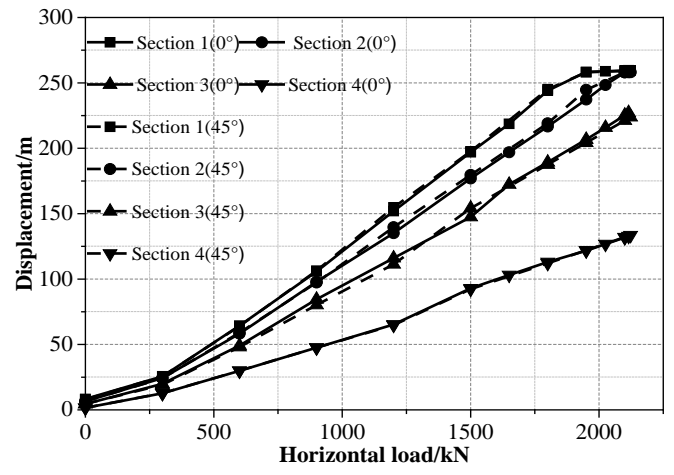

(c) Tensile side of the inner steel tube.

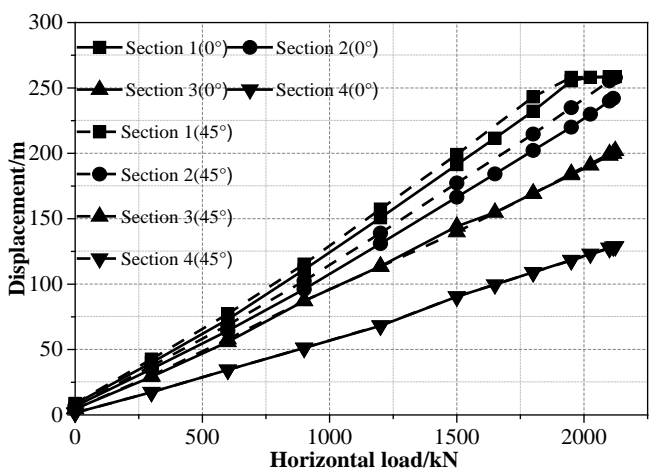

(b) Compressed side of the outer steel tube.

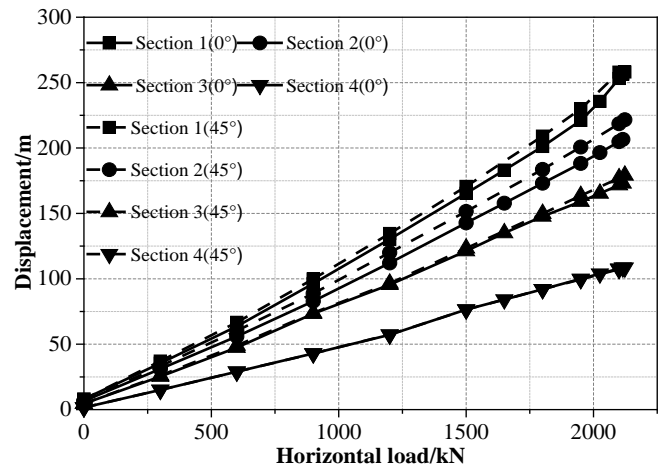

(d) Compressed side of the inner steel tube.

Figure 14 Stresses in the lower position of each tower section under conditions of different loading directions.

Because the longitudinal ribs are not set in the tower sections 3 and 4, the stresses are basically same when the loads are applied in these two directions. In the tower sections 1 and 2 with the longitudinal ribs, a difference is shown in the growth of stress, but not significant. It can be considered that the changes of relative position of loading directions and the ribs have little influence on stress. Therefore, in the design of the tower, there is no need to take the position relationship between the longitudinal ribs and loading directions into consideration.

\section{Conclusions}

This research mainly discusses the influences of the annular and longitudinal ribs used during the segmented assembly of the tower on the mechanical performances of the tower.

1. The annular ribs slightly affect the flexibility of the tower before yielding and the enhancement effects are extremely limited, which is shown in the displacement-load curve. The 
largest influences of the existence of the annular ribs lie in the influences on the stress level in the inner and outer steel tubes in the tower. In the tensile side, concrete cannot provide sufficient support for the annular ribs, so the stress distribution in the inner and outer steel tubes is slightly adjusted. In the compressive zone, owing to the compressive performance, the concrete is not only able to bear loads, but also sufficiently constrains the annular ribs, so that slippage between the outer steel tube connected with the annular ribs and concrete greatly decreases, while stress increases. In the inner steel tube, the stress reduces because more internal forces are put on the outer steel tube. Therefore, the outer steel tube in the compressed side yields in advance and then is damaged due to the existence of the annular ribs, which should be considered in the design of the tower structure.

2. The existence of longitudinal ribs insignificantly enhances the deformation resistance of the tower and mildly changes stress in the inner and outer steel tubes. There are many reasons for this. Firstly, the longitudinal ribs occupy a smaller area in the cross section of the tower compared with the inner and outer steel tubes, so they are not able to greatly improve the carrying capacity of the tower. Secondly, the longitudinal ribs are added based on the annular ribs. Because the annular ribs have greatly changed the stress level of the tower, the influences of the longitudinal ribs on this basis are not obvious. Furthermore, slippage contact is adopted between concrete and the longitudinal ribs, so enough constraints cannot be put on the longitudinal ribs just as those applied on the annular ribs.

3. The impacts of the longitudinal ribs on the displacement and stress of the tower are not highly correlated to the relative position of the direction for applying loads and the longitudinal ribs. This study discusses the changes of displacement and stress under conditions that the loads are applied in directions parallel to the ribs and showing an angle of $45^{\circ}$ with the ribs. The results demonstrate that when loading in these two conditions, the curves of displacement growth are basically overlapped. Moreover, the consistent stresses are generated in the tensile and compressed sides of the inner and outer steel tubes. Therefore, the effects of longitudinal ribs on the tower do not change with their relative position with the loading direction.

\section{References}

[1] Holmes, J.D. (1994) Along-Wind Responses of Lattice Tower: Part I-Derivation of Expressions for Gust Response Factors. Engineering Structures, 16(4), 287.

[2] Ryan, K. and Jason, C. (2000) FEMA 356 the Seismic Rehabilitation of Buildings. Federal Emergency Management Agency, 23(3), 4-6.

[3] Jimeno, J. (2012) Concrete Towers for Multi-Megawatt Turbines. Wind Systems, 2012, 40-45.

[4] Serna, J. and Jimeno, J. (2010) Precast Concrete Wind Towers - the Rise to the Next Level in Hubheight and Support Capacity for Large Turbines. Modern Energy Review, 2(2), 54-59.

[5] Qu, C.Z., Wang, Q. and Xiong, X.Z. (2013) Finite Element Analysis of Concrete Filled Double Skin Steel Tube for Wind Turbine Tower. Electric Power Construction, 34(12), 44-47.

[6] Dong, C.H. (2015) Research on Mechanical Performance of Steel-Concrete Composite Structure for Wind Turbine Tower [Master Thesis]. Weihai: Harbin Institute of Technology (Weihai) (in Chinese). 